\title{
The pan-deacetylase inhibitor panobinostat modulates the expression of epithelial-mesenchymal transition markers in hepatocellular carcinoma models
}

\author{
PIETRO DI FAZIO $^{1 *}$, ROBERTA MONTALBANO $^{1 *}$, KARL QUINT $^{1}$, BEATE ALINGER $^{2}$, RALF KEMMERLING $^{2}$, \\ TOBIAS KIESSLICH ${ }^{2}$, MATTHIAS OCKER ${ }^{1 * *}$ and DANIEL NEUREITER ${ }^{2 * *}$ \\ ${ }^{1}$ Institute for Surgical Research, Philipps-University Marburg, Baldingerstrasse, Marburg 35043, Germany; \\ ${ }^{2}$ Institute of Pathology, Paracelsus Medical University/Salzburger Landeskliniken (SALK), Salzburg 5020, Austria
}

Received June 11, 2012; Accepted September 10, 2012

DOI: $10.3892 / \mathrm{ol} .2012 .951$

\begin{abstract}
Deacetylase inhibitors (DACis) represent a novel therapeutic option for human cancers by classically affecting proliferation or apoptosis. Since transdifferentiation and dedifferentiation play a key role in carcinogenesis, we investigated the epigenetic influence on the molecular differentiation status in human hepatocellular carcinoma (HCC) models. Markers of differentiation, including cytokeratin (Ck) 7 , Ck8, Ck18, Ck19, Ck20, vimentin, sonic hedgehog homolog (SHH), smoothened (Smo), patched (Ptc), glioma-associated oncogene homolog 1 (Gli1), CD133, octamer-binding transcription factor 4 (Oct4) and $\beta$-catenin, were examined in the human HCC cell lines HepG2 and Hep3B in vitro and in vivo (xenograft model) using quantitative real-time PCR and immunohistochemistry following treatment with the pan-DACi panobinostat (LBH589). Compared to untreated controls, treated HepG2 xenografts, and to a lesser extent cell lines, demonstrated a significant increase of differentiation markers Ck7 and Ck19 (classical cholangiocellular type) and Ck8 and Ck18 (classical HCC type), and a decreased level of dedifferentiation markers vimentin (mesenchymal) and
\end{abstract}

Correspondence to: Dr Daniel Neureiter, Institute of Pathology, Paracelsus Medical University/Salzburger Landeskliniken (SALK), 48 Müllner Hauptstrasse, Salzburg 5020, Austria

E-mail:d.neureiter@salk.at

${ }^{*}$ Contributed equally

***oint senior authorship

Abbreviations: BW, body weight; CTGF, connective tissue growth factor; Ck, cytokeratin; DACi, deacetylase inhibitor; Ptc, patched; Smo, smoothened; EMT, epithelial-mesenchymal transition; HCC, human hepatocellular carcinoma; SHH, sonic hedgehog homolog

Key words: inhibitors of deacetylases, hepatocellular carcinoma, epigenetics, transdifferentiation, dedifferentiation, epithelial-mesenchymal transition
SHH/Ptc (embryonic), paralleled with a more membranous expression of $\beta$-catenin. These findings were dose-dependently correlated with tumor size, necrosis rate, microvessel density and mitosis/Ki-67-associated proliferation rate. Our results demonstrate that the differentiation status of human HCC cells is influenced by the pan-DACi panobinostat, indicating that this treatment may influence the epithelial-mesenchymal transition (EMT) status related to metastasis and aggressiveness.

\section{Introduction}

Deacetylase inhibitors (DACis) represent novel therapeutic options for human cancer. As previously demonstrated by our group $(1,2)$, DACis, as epigenetic modulators, have promising results in various human cancer types. The antitumor efficacy of DACis has been extensively studied with respect to their antiproliferative and proapoptotic activity in a number of cancers $(3,4)$, and additional effects of DACis have now been identified that act through alternative mechanisms of cell demise $(5,6)$.

With the exception of proliferation and apoptosis, the status of transdifferentiation and dedifferentiation appears to play a key role in tumor initiation, progression and metastasis. Transdifferentiation defines the process of converting one differentiated cell type into another (7-9), while dedifferentiation defines the phenomena of reexpressing genes related to embryonic development and stem cell characteristics (10). The ability of cells to transdifferentiate and dedifferentiate plays a key role in invasion and metastasis by the process of epithelial-mesenchymal-transition (EMT) $(11,12)$. This phenomenon refers to a number of important mechanisms within current carcinogenesis models and has been fully integrated in the updated 'Hallmarks of cancer' by Hanahan and Weinberg $(13,14)$.

Liver cells are characterized by a genetic pattern conferring them the capability to extensively regenerate following liver resection and differentiation during chronic inflammatory conditions. Due to these properties, liver cells may undergo neoplastic transformations, rendering liver cancer as the second and sixth most common cause of cancer-related mortality 
in males and females, respectively (15). Although several compounds with various modes of action have been recently introduced for liver cancer treatment, the overall response rates remain dissatisfactory (16). In recent years, DACis revealed a strong efficacy in the treatment of liver cancer as well as other solid and hematopoietic malignancies (2). Since EMT is extremely well-characterized in the liver $(17,18)$ and the knowledge of epigenetic regulation in EMT is growing (19), the influence of DACis should be investigated (20).

Our earlier studies on human liver (21), biliary tract (22) and pancreatic cancer cell lines (23) revealed various morphological patterning in association with the molecular expression of differentiation in xenograft models. We previously detected altered patterns of differentiation in pancreatic cancer models following treatment with the histone deacetylase (HDAC) inhibitor SAHA and the methyltransferase inhibitor zebularine (24). The cinnamic hydroxamic acid pan-DACi panobinostat (LBH589) is a novel potent inhibitor of all HDAC enzymes (25) and has already entered clinical development, particularly for hematological diseases including multiple myeloma, Hodgkin's lymphoma and AML (26).

Here, we investigate the influence of panobinostat on the differentiation status in human hepatocellular carcinoma (HCC) cell lines (27). We analyzed the expression of transdifferentiation markers including cytokeratin (Ck) 7, Ck8, Ck18, Ck19 and $\mathrm{Ck} 20$, and dedifferentiation markers including $\beta$-catenin, vimentin, members of the hedgehog pathway, Oct 4 and CD133, in vitro and in vivo (in xenografts).

\section{Material and methods}

Cell culture. Human HCC cell lines HepG2 (p53wt) and Hep3B (p53null) were cultured under standard conditions as described in a previous study (27). Cells were treated with $0.1 \mu \mathrm{M}$ panobinostat, kindly provided by Novartis Pharma AG (Basel, Switzerland) and prepared as described previously (27), and analyzed or processed for further experiments after 6-72 h. Animal experiments complied with the institute's guidelines and were approved by the Government of Lower Franconia (Würzburg, Germany) prior to the experiment.

Xenograft specimens. Formalin-fixed and paraffin-embedded specimens of HepG2 xenografts treated daily with intraperitoneal panobinostat injections of $2.5 \mathrm{mg} / \mathrm{kg}$ of body weight (BW) or $10 \mathrm{mg} / \mathrm{kg}$ of BW were obtained from a previous study (27).

Quantitative real-time reverse-transcription polymerase chain reaction (RT-PCR). For quantitative real-time RT-PCR analysis of mRNA using SYBR-Green detection, total cellular RNA was extracted using the RNeasy mini kit (Qiagen, Hilden, Germany) according to the manufacturer's instructions, and reverse transcription (RT) was conducted using the QuantiTect Reverse Transcription kit (Qiagen). QuantiTect primers for human Ck7 (NM_005556), Ck8 (NM_002273), Ck18 (NM_000224), Ck19 (NM_002276), vimentin (NM_003380), $\beta$-catenin (NM_001904), sonic hedgehog homolog (SHH) (NM_000193), patched (Ptc) (NM_000264), smoothened (Smo) (NM_005631), glioma-associated oncogene homolog 1 (Gli1) (NM_005269), Oct4 (NM_203289), CD133 (NM_006017) and glyceralde- hyde 3-phosphate dehydrogenase (GAPDH) (NM_002046) were purchased from Qiagen and run with the SsoFast ${ }^{\mathrm{TM}}$ EvaGreen Supermix (Bio-Rad Laboratories GmbH, Munich, Germany) on a CFX96 ${ }^{\mathrm{TM}}$ real-time PCR detection system (Bio-Rad Laboratories GmbH). Results were analyzed using the CFX Manager version 2.0 and Rest 2008 software, which was normalized to GAPDH mRNA content for each sample.

Tissue preparation and immunohistochemistry. All specimens were fixed in $10 \%$ buffered formalin, routinely processed and embedded in paraffin wax. Immunohistochemistry was conducted using routine diagnostic methods as recently published (28), and immunohistochemical stainings were obtained using an autostainer system (Dako, Glostrup, Denmark) according to the manufacturer's instructions. Antigen retrieval was conducted by heat induced epitope retrieval in antigen retrieval buffer ( $\mathrm{pH} 9.0$ ) (Dako) at $95^{\circ} \mathrm{C}$ for $40 \mathrm{~min}$. The primary antibodies for $\mathrm{Ck} 7, \mathrm{Ck} 8, \mathrm{Ck} 18, \mathrm{Ck} 19$, Ck20, vimentin, $\mathrm{SHH}$, Ptc and $\beta$-catenin were used to clarify the differentiation status (type of antibodies, vendors, pretreatment conditions and dilutions) as previously published (21). Additionally, primary polyclonal rabbit antibodies of connective tissue growth factor (CTGF; 1:50 dilution; $\mathrm{pH}$ 9; antigen retrieval; Abcam, Cambridge, UK) were used to characterize EMT interactions. Tonsils and lymph nodes served as positive controls. Negative control experiments were performed using phosphate-buffered saline in place of the primary or secondary antibodies and the same processing as previously described (not shown).

Interpretation of immunohistochemistry. The stained slides were digitalized using the ImageAccess 11 Enterprise software (Imagic Bildverarbeitung, Glattbrugg, Switzerland). The percentage of positive cells (extensity) was detected by evaluating the images per high-power field (HPF; magnification, $\mathrm{x} 400$ ) using the particle analysis module with optimized binarization method. The level of staining intensity $(0$, none; 1, low; 2, moderate; and 3, strong) was assessed by two independent pathologists (R.K. and D.N.). Finally, an expression score was calculated by multiplying the extensity and intensity score (22).

Statistical analysis. Statistical analysis was conducted using SPSS 18.0 (SPSS Inc., Chicago, IL, USA). Univariate analysis of variance (ANOVA) was used to test for differences between the groups of tissue samples using the least significant difference (LSD) post-hoc test to adjust for multiple comparisons. The Pearson's product-moment correlation coefficient test was used to measure correlation. $\mathrm{P}<0.05$ was considered to indicate a statistically significant difference.

\section{Results}

In vitro expression pattern of transdifferentiation and dedifferentiation markers following panobinostat treatment. As shown in Fig. 1, treatment with panobinostat markedly decreased the mRNA level of $\mathrm{Ck} 7$ in Hep3B after $6 \mathrm{~h}$ and in HepG2 after 48 h. Ck8 and Ck18 transcripts revealed unpredictable patterns demonstrating no correlation during the time points of both cell lines, while mRNA levels 

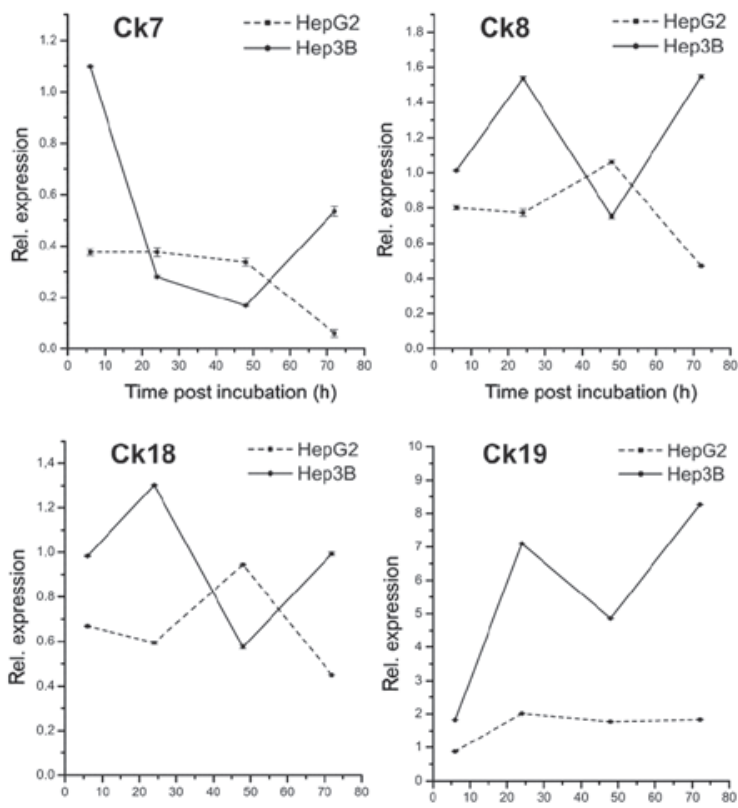

Time post incubation (h)
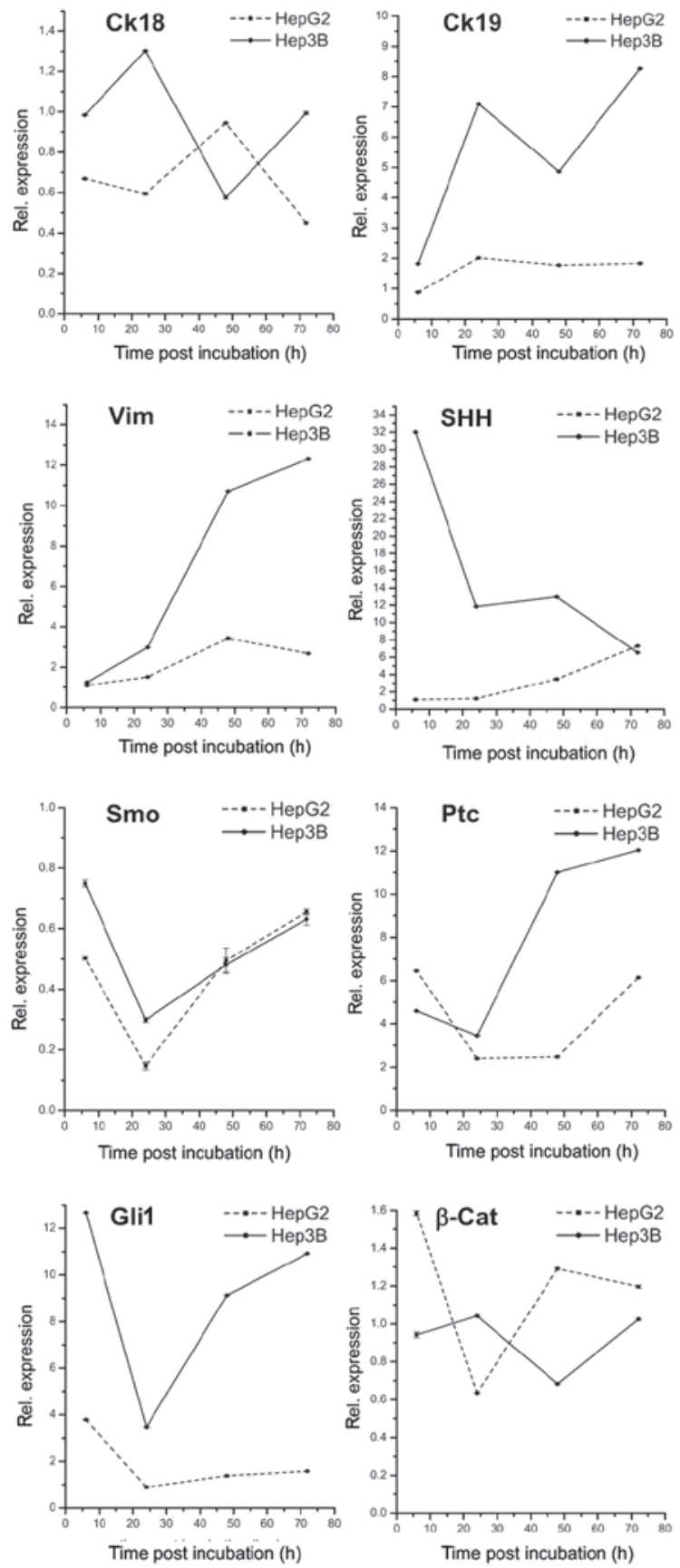

Figure 1. mRNA expression patterns of transdifferentiation and dedifferentiation markers in human hepatic cancer cell lines HepG2 and Hep3B in vitro at $6,24,48$ and $72 \mathrm{~h}$ after $0.1 \mu \mathrm{mol}$ panobinostat treatment. mRNA expression was normalized to GAPDH and all results are expressed relative to untreated controls set at 1.0. Results are expressed as mean \pm SEM of three independent experiments conducted in triplicate. Ck, cytokeratin; Vim, vimentin; SHH, sonic hedgehog homolog; Ptc, patched; Smo, smoothened; Gli1, glioma-associated oncogene homolog 1 ; $\beta$-Cat, $\beta$-catenin; GAPDH, glyceraldehyde 3-phosphate dehydrogenase; SEM, standard error of mean. of Ck19 increased early in both cell lines. The level of vimentin mRNA significantly increased in both cell lines with a marked upregulation after $48 \mathrm{~h}$. With regards to the hedgehog pathway components, a heterogeneous expression pattern was observed over the treatment time periods: mRNA of SHH significantly increased in HepG2 cells, while the opposite effect was observed in Hep3B cells. Transcript levels of Smo were extremely low in both cell lines with a stable decrease occurring after $72 \mathrm{~h}$. In contrast to Smo, Ptc and Gli transcripts were highly expressed and revealed the highest expression 6 and $72 \mathrm{~h}$ after panobinostat treatment. Overall, the mRNA levels of $\beta$-catenin were stable in HepG2 cells with a slight decrease observed $24 \mathrm{~h}$ after treatment, and were stable in Hep3B cells with a slight decrease observed $48 \mathrm{~h}$ after panobinostat treatment. Finally, Oct4 was not detectable in either cell line, and CD133 revealed low expression in Hep3B cells and no expression in HepG2 cells (data not shown).

Correlation analysis of transdifferentiation and dedifferentiation markers in vitro. Post-treatment correlation analysis (Table I) between differentiation markers in HepG2 cells analyzed over time revealed a significant inverse correlation between $\mathrm{Ck} 7$ and $\mathrm{SHH}$ markers as well as $\mathrm{Ck} 19$ and Gli markers $(\mathrm{P}<0.001)$. A heterogeneous correlative association was found between all investigated markers, whereby the majority of transdifferentiation markers were negatively associated with markers of dedifferentiation (e.g. Ck7 vs. vimentin and Ck8/Ck18/Ck19 vs. Smo or Ptc).

Correlation analysis for Hep3B cells revealed overall positive correlations between markers of dedifferentiation. Notably, a positive correlation of $\mathrm{Cks}$ to $\beta$-catenin was also identified. Comparable to HepG2 cells, the majority of transdifferentiation markers were negatively associated with markers of dedifferentiation, whereby the statistical analysis of vimentin mRNA were diametric to HepG2.

In vivo expression pattern of transdifferentiation and dedifferentiation markers following panobinostat treatment. The investigated HCC xenografts displayed a predominantly solid growth pattern. As previously published, proliferation and apoptosis levels were significantly decreased and increased, respectively, in xenografts treated with panobinostat (27).

mRNA levels of Cks were also evaluated in HepG2 xenografts 4 weeks after treatment with $10 \mathrm{mg} / \mathrm{kg}$ BW panobinostat. As shown in Fig. 2, panobinostat caused a marked transient reduction of $\mathrm{Ck} 7 \mathrm{mRNA}$ transcript after 1 week, and restored it to the basal level after 4 weeks. In comparison, the mRNA of Ck19 was stably expressed and revealed an upregulation 4 weeks after panobinostat treatment. Panobinostat induced similar effects on the Ck8 and Ck18 levels; both were upregulated after 2 weeks but were slightly reduced after 4 weeks of treatment. Notably, vimentin mRNA was significantly decreased after 1 week, with exorbitant increase 2 and 4 weeks following panobinostat treatment. We also investigated the mRNA expression pattern of hedgehog pathway components and revealed a notable decrease of all investigated members of the pathway (SHH, Smo, Ptc and Gli), which were no longer detectable after 4 weeks of treatment with panobinostat. The mRNA of $\beta$-catenin was stable until the second week, and increased slightly after that. Finally, mRNA 
Table I. Correlation analysis of transdifferentiation and dedifferentiation markers following panobinostat treatment in vitro.

\begin{tabular}{|c|c|c|c|c|c|c|c|c|c|c|}
\hline & Ck8 & Ck18 & Ck19 & Vim & $\mathrm{SHH}$ & Smo & Ptc & Gli1 & CD133 & $\beta$-Cat \\
\hline \multicolumn{11}{|l|}{$\mathrm{Ck} 7$} \\
\hline HepG2 & 0.77 & 0.57 & -0.31 & -0.42 & $-0.96^{\mathrm{a}}$ & -0.68 & -0.48 & 0.21 & n.a. & -0.06 \\
\hline Нep3B & -0.05 & 0.18 & -0.64 & -0.52 & 0.81 & 0.83 & -0.35 & 0.69 & $0.96^{\mathrm{a}}$ & 0.33 \\
\hline \multicolumn{11}{|l|}{ Ck8 } \\
\hline HepG2 & & $0.99^{\mathrm{a}}$ & -0.12 & 0.23 & -0.58 & -0.30 & -0.63 & 0.00 & n.a. & 0.14 \\
\hline Нер3B & & 0.83 & 0.74 & -0.01 & -0.48 & -0.27 & -0.16 & -0.41 & -0.29 & $0.91^{\mathrm{a}}$ \\
\hline \multicolumn{11}{|l|}{ Ck18 } \\
\hline HepG2 & & & -0.11 & 0.43 & -0.37 & -0.07 & -0.55 & 0.00 & n.a. & 0.27 \\
\hline Hep3B & & & 0.32 & -0.56 & -0.02 & -0.30 & -0.68 & -0.50 & 0.04 & $0.92^{\mathrm{a}}$ \\
\hline \multicolumn{11}{|l|}{ Ck19 } \\
\hline HepG2 & & & & 0.52 & 0.39 & -0.33 & -0.65 & $-0.99^{a}$ & n.a. & -0.82 \\
\hline Hep3B & & & & 0.58 & $-0.95^{\mathrm{a}}$ & -0.52 & 0.39 & -0.51 & -0.83 & 0.39 \\
\hline \multicolumn{11}{|l|}{ Vim } \\
\hline HepG2 & & & & & 0.64 & 0.44 & -0.34 & -0.53 & n.a. & 0.05 \\
\hline Нер3B & & & & & -0.74 & 0.01 & $0.97^{\mathrm{a}}$ & 0.18 & -0.61 & -0.34 \\
\hline \multicolumn{11}{|l|}{ Shh } \\
\hline HepG2 & & & & & & 0.72 & 0.32 & -0.31 & n.a. & 0.10 \\
\hline Нер3B & & & & & & 0.57 & -0.57 & 0.48 & 0.94 & -0.07 \\
\hline \multicolumn{11}{|l|}{ Smo } \\
\hline HepG2 & & & & & & & 0.68 & 0.38 & n.a. & 0.76 \\
\hline Нер3B & & & & & & & 0.22 & $0.97^{\mathrm{a}}$ & 0.78 & -0.01 \\
\hline \multicolumn{11}{|l|}{ Ptc } \\
\hline HepG2 & & & & & & & & 0.74 & n.a. & 0.65 \\
\hline Нер3B & & & & & & & & 0.40 & -0.41 & -0.42 \\
\hline \multicolumn{11}{|l|}{ Gli } \\
\hline HepG2 & & & & & & & & & n.a. & 0.82 \\
\hline Нер3В & & & & & & & & & 0.67 & -0.21 \\
\hline \multicolumn{11}{|l|}{ CD133 } \\
\hline HepG2 & & & & & & & & & & n.d. \\
\hline Нер3В & & & & & & & & & & n.d. \\
\hline
\end{tabular}

Pearson's correlation coefficient was used to measure the correlation between mRNA expression. ${ }^{\mathrm{a}} \mathrm{P}<0.01$. Ck, cytokeratin; Vim, vimentin; SHH, sonic hedgehog homolog; Smo, smoothened; Ptc, patched; Gli1, glioma-associated oncogene homolog 1; $\beta$-Cat, $\beta$-catenin. n.a., not applicable; n.d., not determined.

levels of Oct4 and CD133 were not detectable at any time point in vivo (data not shown).

HepG2 xenografts implanted in nude mice treated with $10 \mathrm{mg} / \mathrm{kg}$ BW panobinostat demonstrated a significant increase of $\mathrm{Ck} 7$ protein levels compared with control xenografts (vehicle only; Fig. 3). Ck8 and Ck18 also demonstrated a significant increase following treatment with $10 \mathrm{mg} / \mathrm{kg}$ BW panobinostat in contrast to vimentin, a mesenchymal marker, which was significantly decreased. A lower dose of panobinostat $(2.5 \mathrm{mg} / \mathrm{kg} \mathrm{BW})$ was able to induce a significant increase of $\mathrm{Ck} 19$ protein expression; while the protein expression of $\mathrm{Ck} 20$ minimally increased following panobinostat treatment at high dose $(<1$ cell/HPF; data not shown). Immunostaining for $\beta$-catenin revealed a different cellular distribution, highlighting a reduction of its cytoplasmic level and a slight increase of its cellular membrane localization, particularly following treatment with $10 \mathrm{mg} / \mathrm{kg}$ BW panobi- nostat. Although the expression levels of hedgehog pathway members SHH and Ptc were low in the untreated control xenografts, a significant reduction of the protein levels was observed, particularly following treatment with $10 \mathrm{mg} / \mathrm{kg} \mathrm{BW}$ panobinostat.

Correlation analysis of transdifferentiation and dedifferentiation markers in vivo. As listed in Table II, Cks as markers of transdifferentiation demonstrated a constant, partly significant, negative correlation with proliferation rate (Ki-67), mitosis rate, microvessel density, size of tumor and necrosis, indicating an inverse temporal correlation following panobinostat treatment. Overall, the most significant correlations were identified between markers of differentiation and the rate of mitosis and microvessel density. We revealed that those morphological parameters were negatively associated with the expression of Ck7, Ck8/18 and Ck19, but positively associated with markers 

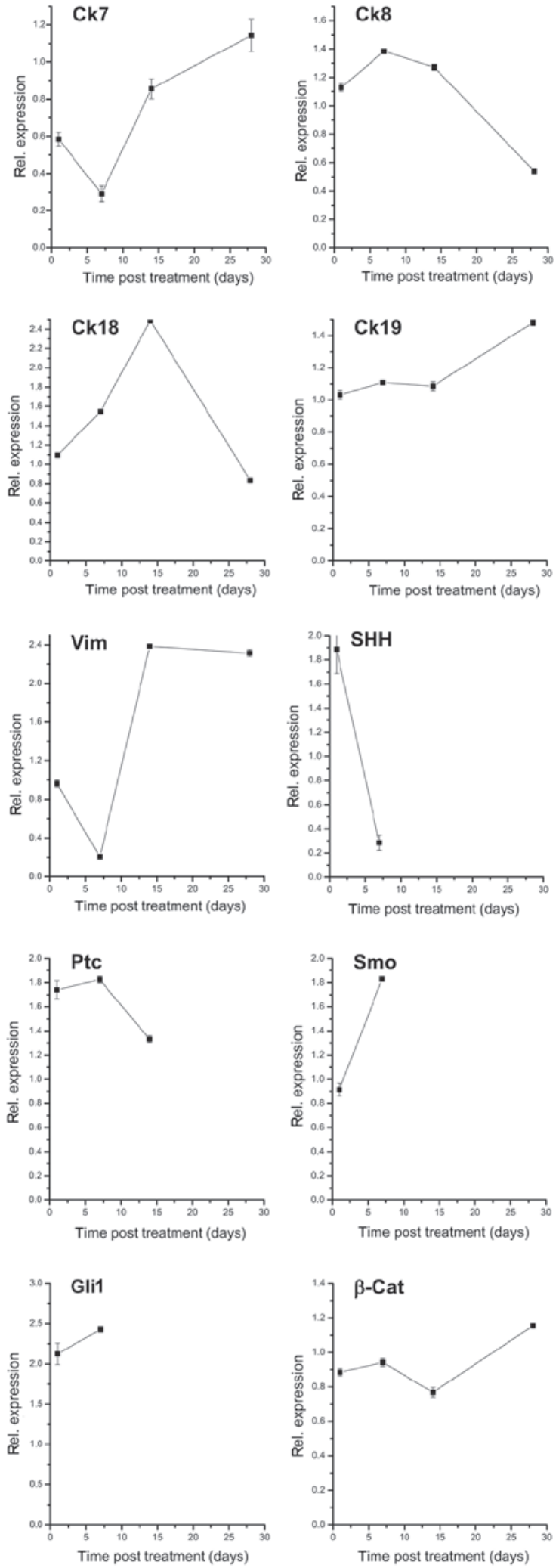

Figure 2. mRNA expression pattern of transdifferentiation and dedifferentiation markers under $10 \mathrm{mg} / \mathrm{kg}$ BW panobinostat in HepG2 xenografts at 1 day, 1 week, 2 weeks and 4 weeks after treatment. Where values are missing, mRNA could not be detected. mRNA expression was normalized to GAPDH and all results are expressed relative to untreated controls set at 1.0 Results are expressed as mean \pm SEM of three independent experiments conducted in triplicate. Ck, cytokeratin; Vim, vimentin; SHH, sonic hedgehog homolog; Ptc, patched; Smo, smoothened; Gli1, glioma-associated oncogene homolog $1 ; \beta$-Cat, $\beta$-catenin; BW, body weight; GAPDH, glyceraldehyde 3-phosphate dehydrogenase; SEM, standard error of mean.
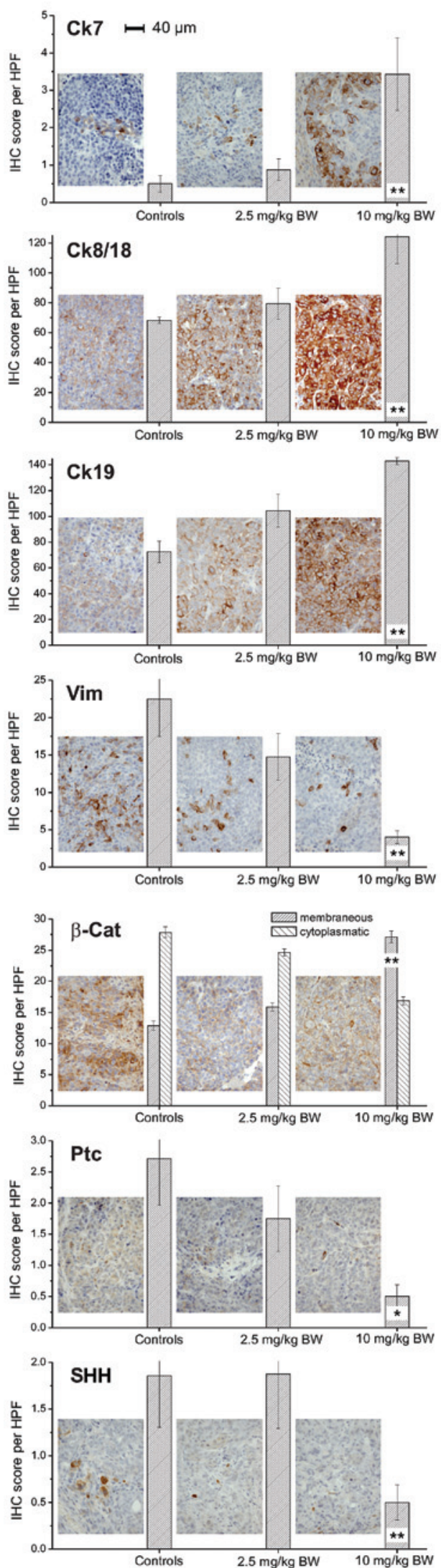

Figure 3. Typical immunohistochemistry protein expression patterns of transdifferentiation and dedifferentiation markers in $\mathrm{HepG} 2$ xenografts 4 weeks after treatment with 2.5 and $10 \mathrm{mg} / \mathrm{kg}$ BW panobinostat. Numerical results are expressed as mean \pm SEM per high-power field (magnification, $x 400$ ) based on the product of intensity and extensity levels. ${ }^{*} \mathrm{P}<0.05$ vs. control; ${ }^{* *} \mathrm{P}<0.01$ vs. control. Ck, cytokeratin; IHC, immunohistochemistry; BW, body weight; SHH, sonic hedgehog homolog; Vim, vimentin; SEM, standard error of mean. 
Table II. Correlation analysis of transdifferentiation and dedifferentiation markers following panobinostat treatment in vivo (HepG2 xenografts).

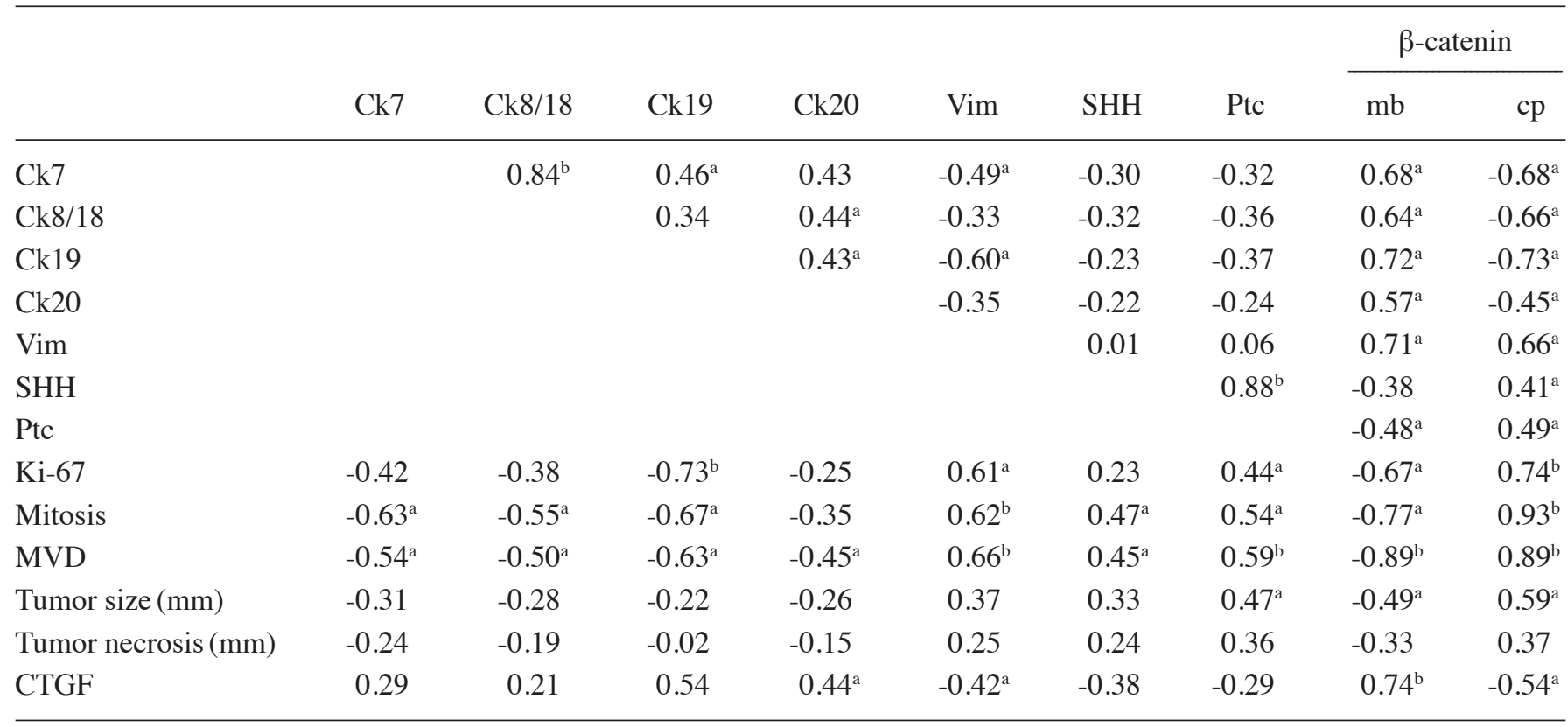

Pearson's correlation coefficient was used to measure the correlation between protein expression. ${ }^{a} \mathrm{P}<0.05$; ${ }^{\text {b }}<0.01$. Ck, cytokeratin; Vim, vimentin; SHH, sonic hedgehog homolog; Ptc, patched; mb, membraneous; cp, cytoplasmic; MVD, microvessel density; CTGF, connective tissue growth factor.

of dedifferentiation, including vimentin and members of the hedgehog pathway. In line with these results, membranous and cytoplasmic levels of $\beta$-catenin demonstrated an inverse correlation pattern; changes of membranous $\beta$-catenin are positively correlated with changes of $\mathrm{Ck}$ expression, while cytoplasmic levels of $\beta$-catenin are inversely correlated with changes of $\mathrm{Ck}$ expression. Finally, the expression of Ck19, Ck20 and membranous $\beta$-catenin were significantly associated with CTGF expression, while an opposite correlation was identified for the expression of vimentin and cytoplasmic $\beta$-catenin.

\section{Discussion}

We demonstrated that with the exception of proliferation and apoptosis (27) the differentiation status of human HCC cell lines in vitro and in vivo (using a xenograft model) may be influenced by the pan-DACi panobinostat (LBH589). This suggests that DACis may also influence cellular processes of differentiation and EMT transition, leading to the reversion of a malignant, undifferentiated phenotype into a more benign and differentiated phenotype.

As discussed by a number of authors, the processes of differentiation, transdifferentiation and dedifferentiation are essentially involved in the cellular development, maintenance and regeneration of different biological systems (7-9).

In the last few years, the impact of differentiation in carcinogenesis has gained increasing attention and it has been included as a basic mechanism in the cancer stem cell model (14). Tumor-specific stem cell signatures were proven experimentally (29), predicting clinical endpoints including time of tumor progression or outcome (30). Here, we reveal that untreated human HCC cell lines (HepG2 and Hep3B) express markers of dedifferentiation rather than of transdifferentiation. Differences between the in vitro and in vivo situation were identified, which may be induced by the effect of extracellular matrix interactions in the xenograft model (21) since matrix stiffness influences proliferation and chemotherapeutic resistance as well as cellular dormancy and stem cell characteristics in HCC (31). Notably, CD133 expression was only observed in Hep3B cells, which confirms the results of a recent study revealing that CD133 expression levels are associated with the upregulation of invasion- and EMT-associated genes leading to greater cell migration in various human hepatic cancer cell lines (32). This reflects the whole differentiation capacity of human liver cancer $(17,18)$, which should be the concept for further experimental investigations and the new future approach for patient risk stratification and therapeutic decision, as it has assumed strong relevance in other solid malignancies including breast cancer $(33,34)$.

Until now, the therapeutic effectiveness following chemotherapy and surgical tumor resection is routinely evaluated by investigating the tumor volume and the signs of regression. The estimation of tumor volume or its reduction is semi-quantitatively assessed as previously reviewed (35), and the signs of regression include tumor cell pyknosis, cytoplasmic vacuolization/fragmentation and necrosis. Comparable to our previous study, we identified that tumor size and tumor necrosis of the xenografts decreased and increased, respectively, following panobinostat treatment (27), which was recently confirmed in combination with sorafenib (36). Other approaches characterized the tumor-associated inflammation to identify therapy-associated prognostic factors (37). Nevertheless, it is worth characterizing the tumor differentiation status since it is known that tumors change their morphological and molecular 
phenotype due to the onset of chemotherapy resistance or by shifting the differentiation status and selecting specific tumor subgroups including tumor stem cells (38). Gastrointestinal stromal tumors present different phenomena of transdifferentiation and dedifferentiation, including loss of c-kit expression or rhabdomyoid differentiation following targeted therapy with kinase inhibitors (imatinib mesylate) (39). Analysis of breast cancer following neoadjuvant chemotherapy revealed a different expression of the estrogen and progesterone receptors compared to untreated cancer (40). In contrast to classical $\mathrm{Ck}$ profiling, these markers are not lineage- or differentiation-specific, demonstrating only an on-off switch phenomenon of activation inside these tumor cells (41). Additionally, hematopoietic cancer cells revealed a loss of their surface markers, including CD20, following target therapy with retuximab, a specific antibody against CD20 (42). Furthermore, treatment of MDS and AML with the DNA methylation inhibitor decitabine vidaza induced different morphological changes, including colony formation, and expression of hematopoietic differentiation markers (43).

Our data demonstrates that human liver cancer cell lines change their status shifting from a dedifferentiated acquired pattern to a well-differentiated status following treatment with panobinostat. These results are in line with earlier studies in a human pancreatic cancer cell model using a combination of the histone deacetylase inhibitor SAHA and the methyltransferase inhibitor Zebularine (24). The effect of panobinostat demonstrated a time- and dose-dependent activity (particularly in vitro), whereby heterogeneous effects were observed in vitro and in vivo as previously discussed. Overall, we identified that treatment with panobinostat not only caused a decrease of dedifferentiation markers, but also an increase of differentiation markers including $\mathrm{Cks}$. This supports the idea of a well-differentiated pattern having a similar morphological and molecular plasticity of human liver cells and preventing the EMT in the process of tumor migration and metastasis $(11,12)$. Further experiments using invasion assays are required to support this hypothesis. Notably, the expression pattern of differentiation could significantly be linked to the expression of CTGF contributing to HCC cell dedifferentiation (44). Therefore, differentiation patterning may be used as an additional prognostic and predictive indicator for therapeutic effectiveness as recently discussed (45). We could demonstrate that the expression of markers, including $\mathrm{Cks}$, vimentin and $\beta$-catenin, correlates with the effectiveness of photodynamic therapy (46) or experimental Wnt pathway inhibitors $(22,47)$ in a biliary tract cancer model. Additionally, the importance of differentiation status is supported by our results, which demonstrate that markers of differentiation are significantly inversely correlated to classical prognostic tumor markers, including tumor size, tumor necrosis, mitosis rate, microvessel density and $\mathrm{Ki}-67$-associated proliferation rate in the xenograft model. This should be taken into account since targeted therapies are often underestimated by conventional parameters like RECIST or WHO radiology. Therefore, functional biomarkers like differentiation markers should be established.

Finally, we are aware that we descriptively present the effect of the pan-DACi panobinostat on EMT in a human liver cancer model, which should be proven and supported by further functional investigations using siRNA or small molecules interacting with intermediary filaments $(48,49)$.

In conclusion, the pan-DACi panobinostat influences not only the classical markers of cancerogenesis including proliferation and apoptosis, but also the differentiation status of human hepatic cancer cell lines HepG2 and Hep3B. Since the epigenetic-associated shift of differentiation is paralleled by morphological markers such as tumor size and proliferation, more effort should be focused on the differentiation status of tumors in order to provide additional information for therapeutic effectiveness and success.

\section{Acknowledgements}

The expert technical assistance of Mrs Berta Lechner is gratefully acknowledged. T.K. was supported by a research grant from the research fund of the Paracelsus Medical University in Salzburg (Grant No. R-10/04/17-KIE). M.O. was supported by the Wissenschaftlicher Verein der Pathologie Salzburg/ Austria grant of the University Hospital Medical Center Giessen and Marburg (UKGM), as well as a grant from Novartis Pharma GmbH (Germany). Additionally, P.D.F. received a Dame Sheila Sherlock Post-Doctoral Fellowship from the European Association for the Study of the Liver (EASL).

\section{References}

1. Stintzing S, Kemmerling R, Kiesslich T, Alinger B, Ocker M and Neureiter D: Myelodysplastic syndrome and histone deacetylase inhibitors: 'to be or not to be acetylated'? J Biomed Biotechnol 2011: 214143, 2011

2. Miller CP, Singh MM, Rivera-Del Valle N, Manton CA and Chandra J: Therapeutic strategies to enhance the anticancer efficacy of histone deacetylase inhibitors. J Biomed Biotechnol 2011: 514261, 2011.

3. Jana S and Paliwal J: Apoptosis: potential therapeutic targets for new drug discovery. Curr Med Chem 14: 2369-2379, 2007.

4. Schneider-Stock R and Ocker M: Epigenetic therapy in cancer: molecular background and clinical development of histone deacetylase and DNA methyltransferase inhibitors. IDrugs 10: 557-561, 2007.

5. Ellis L, Atadja PW and Johnstone RW: Epigenetics in cancer: targeting chromatin modifications. Mol Cancer Ther 8: 1409-1420, 2009.

6. Ocker M: Deacetylase inhibitors - focus on non-histone targets and effects. World J Biol Chem 1: 55-61, 2010.

7. Jones PA and Taylor SM: Cellular differentiation, cytidine analogs and DNA methylation. Cell 20: 85-93, 1980.

8. Jones PA, Taylor SM and Wilson V: DNA modification, differentiation, and transformation. J Exp Zool 228: 287-295, 1983.

9. Eguchi G and Kodama R: Transdifferentiation. Curr Opin Cell Biol 5: 1023-1028, 1993.

10. Neureiter D, Herold C and Ocker M: Gastrointestinal cancer only a deregulation of stem cell differentiation? (Review). Int J Mol Med 17: 483-489, 2006.

11. Gavert N and Ben-Ze'ev A: Epithelial-mesenchymal transition and the invasive potential of tumors. Trends Mol Med 14: 199-209, 2008.

12. Sabbah M, Emami S, Redeuilh G, Julien S, Prevost G, Zimber A, Ouelaa R, Bracke M, De Wever O and Gespach C: Molecular signature and therapeutic perspective of the epithelial-to-mesenchymal transitions in epithelial cancers. Drug Resist Updat 11: 123-151, 2008.

13. Hanahan D and Weinberg RA: The hallmarks of cancer. Cell 100: 57-70, 2000.

14. Hanahan D and Weinberg RA: Hallmarks of cancer: the next generation. Cell 144: 646-674, 2011.

15. Jemal A, Bray F, Center MM, Ferlay J, Ward E and Forman D: Global cancer statistics. CA Cancer J Clin 61: 69-90, 2011. 
16. Greten TF, Korangy F, Manns MP and Malek NP: Molecular therapy for the treatment of hepatocellular carcinoma. Br J Cancer 100: 19-23, 2009.

17. Choi SS and Diehl AM: Epithelial-to-mesenchymal transitions in the liver. Hepatology 50: 2007-2013, 2009.

18. Rountree CB, Mishra L and Willenbring H: Stem cells in liver diseases and cancer: recent advances on the path to new therapies. Hepatology 55: 298-306, 2012.

19. McDonald OG, Wu H, Timp W, Doi A and Feinberg AP: Genome-scale epigenetic reprogramming during epithelial-to-mesenchymal transition. Nat Struct Mol Biol 18: 867-874, 2011.

20. Marquardt JU, Factor VM and Thorgeirsson SS: Epigenetic regulation of cancer stem cells in liver cancer: current concepts and clinical implications. J Hepatol 53: 568-577, 2010.

21. Jabari S, Meissnitzer M, Quint K, Gahr S, Wissniowski T, Hahn EG, Neureiter D and Ocker M: Cellular plasticity of transand dedifferentiation markers in human hepatoma cells in vitro and in vivo. Int J Oncol 35: 69-80, 2009.

22. Kiesslich T, Alinger B, Wolkersdorfer GW, Ocker M, Neureiter D and Berr F: Active Wnt signalling is associated with low differentiation and high proliferation in human biliary tract cancer in vitro and in vivo and is sensitive to pharmacological inhibition. Int J Oncol 36: 49-58, 2010.

23. Neureiter D, Zopf S, Dimmler A, Stintzing S, Hahn EG, Kirchner T, Herold C and Ocker M: Different capabilities of morphological pattern formation and its association with the expression of differentiation markers in a xenograft model of human pancreatic cancer cell lines. Pancreatology 5: 387-397, 2005.

24. Neureiter D, Zopf S, Leu T, Dietze O, Hauser-Kronberger C, Hahn EG, Herold C and Ocker M: Apoptosis, proliferation and differentiation patterns are influenced by Zebularine and SAHA in pancreatic cancer models. Scand J Gastroenterol 42: 103-116, 2007.

25. Maiso P, Carvajal-Vergara X, Ocio EM, Lopez-Perez R, Mateo G, Gutierrez N, Atadja P, Pandiella A and San Miguel JF: The histone deacetylase inhibitor LBH589 is a potent antimyeloma agent that overcomes drug resistance. Cancer Res 66: 5781-5789, 2006.

26. Atadja P: Development of the pan-DAC inhibitor panobinostat (LBH589): successes and challenges. Cancer Lett 280: 233-241, 2009.

27. Di Fazio P, Schneider-Stock R, Neureiter D, Okamoto K, Wissniowski T, Gahr S, Quint K, Meissnitzer M, Alinger B, Montalbano R, Sass G, Hohenstein B, Hahn EG and Ocker M: The pan-deacetylase inhibitor panobinostat inhibits growth of hepatocellular carcinoma models by alternative pathways of apoptosis. Cellular Oncology 32: 285-300, 2010.

28. Kemmerling R, Stintzing S, Muhlmann J, Dietze $O$ and Neureiter D: Primary testicular lymphoma: A strictly homogeneous hematological disease? Oncol Rep 23: 1261-1267, 2010.

29. Alison MR, Lim SM and Nicholson LJ: Cancer stem cells: problems for therapy? J Pathol 223: 147-161, 2011.

30. Ailles LE and Weissman IL: Cancer stem cells in solid tumors. Curr Opin Biotechnol 18: 460-466, 2007

31. Schrader J, Gordon-Walker TT, Aucott RL, van Deemter M, Quaas A, Walsh S, Benten D, Forbes SJ, Wells RG and Iredale JP: Matrix stiffness modulates proliferation, chemotherapeutic response, and dormancy in hepatocellular carcinoma cells. Hepatology 53: 1192-1205, 2011.

32. Na DC, Lee JE, Yoo JE, Oh BK, Choi GH and Park YN: Invasion and EMT-associated genes are up-regulated in B viral hepatocellular carcinoma with high expression of CD133-human and cell culture study. Exp Mol Pathol 90: 66-73, 2011

33. van 't Veer LJ, Dai H, van de Vijver MJ, He YD, Hart AA, Mao M, Peterse HL, van der KK, Marton MJ, Witteveen AT, Schreiber GJ, Kerkhoven RM, Roberts C, Linsley PS, Bernards R and Friend SH: Gene expression profiling predicts clinical outcome of breast cancer. Nature 415: 530-536, 2002.

34. Perou CM, Sorlie T, Eisen MB, van de RM, Jeffrey SS, Rees CA, Pollack JR, Ross DT, Johnsen H, Akslen LA, Fluge O, Pergamenschikov A, Williams C, Zhu SX, Lonning PE, Borresen-Dale AL, Brown PO and Botstein D: Molecular portraits of human breast tumours. Nature 406: 747-752, 2000.
35. Wittekind C and Tannapfel A: Regression grading of colorectal carcinoma after preoperative radiochemotherapy. An inventory. Pathologe 24: 61-65, 2003.

36. Lachenmayer A, Toffanin S, Cabellos L, Alsinet C, Hoshida Y, Villanueva A, Minguez B, Tsai HW, Ward SC, Thung S, Friedman SL and Llovet JM: Combination therapy for hepatocellular carcinoma: additive preclinical efficacy of the HDAC inhibitor panobinostat with sorafenib. J Hepatol 56: 1343-1350, 2012.

37. Zitvogel L, Kepp O and Kroemer G: Immune parameters affecting the efficacy of chemotherapeutic regimens. Nat Rev Clin Oncol 8: 151-160, 2011.

38. Todaro M,Francipane MG, Medema JP and Stassi G: Colon cancer stem cells: promise of targeted therapy. Gastroenterology 138: 2151-2162, 2010

39. Diaz DM, Hernandez AA, Pereira GS, Jaramillo S, Virizuela Echaburu JA and Gonzalez-Campora RJ: Gastrointestinal stromal tumors: morphological, immunohistochemical and molecular changes associated with kinase inhibitor therapy. Pathol Oncol Res 17: 455-461, 2011.

40. Lee J, Im YH, Lee SH, Cho EY, Choi YL, Ko YH, Kim JH, Nam SJ, Kim HJ, Ahn JS, Park YS, Lim HY, Han BK and Yang JH: Evaluation of ER and Ki-67 proliferation index as prognostic factors for survival following neoadjuvant chemotherapy with doxorubicin/docetaxel for locally advanced breast cancer. Cancer Chemother Pharmacol 61: 569-577, 2008.

41. Moll R, Divo M and Langbein L: The human keratins: biology and pathology. Histochem Cell Biol 129: 705-733, 2008

42. Goteri G, Olivieri A, Ranaldi R, Lucesole M, Filosa A, Capretti R, Pieramici T, Leoni P, Rubini C, Fabris G and Lo ML: Bone marrow histopathological and molecular changes of small B-cell lymphomas after rituximab therapy: comparison with clinical response and patients outcome. Int J Immunopathol Pharmacol 19: 421-431, 2006.

43. Ryningen A, Stapnes C and Bruserud O: Clonogenic acute myelogenous leukemia cells are heterogeneous with regard to regulation of differentiation and effect of epigenetic pharmacological targeting. Leuk Res 31: 1303-1313, 2007.

44. Urtasun R, Latasa MU, Demartis MI, Balzani S, Goni S, Garcia-Irigoyen O, Elizalde M, Azcona M, Pascale RM, Feo F, Bioulac-Sage P, Balabaud C, Muntane J, Prieto J, Berasain C and Avila MA: Connective tissue growth factor autocriny in human hepatocellular carcinoma: oncogenic role and regulation by epidermal growth factor receptor/yes-associated protein-mediated activation. Hepatology 54: 2149-2158, 2011.

45. Hoshida Y, Toffanin S, Lachenmayer A, Villanueva A, Minguez B and Llovet JM: Molecular classification and novel targets in hepatocellular carcinoma: recent advancements. Semin Liver Dis 30: 35-51, 2010.

46. Kiesslich T, Neureiter D, Alinger B, Jansky GL, Berlanda J, Mkrtchyan V, Ocker M, Plaetzer K and Berr F: Uptake and phototoxicity of meso-tetrahydroxyphenyl chlorine are highly variable in human biliary tract cancer cell lines and correlate with markers of differentiation and proliferation. Photochem Photobiol Sci 9: 734-743, 2010.

47. Wachter J, Neureiter D, Alinger B, Pichler M, Fuereder J, Oberdanner C, Di Fazio P, Ocker M, Berr F and Kiesslich T: Influence of five potential anticancer drugs on wht pathway and cell survival in human biliary tract cancer cells. Int J Biol Sci 8: $15-29,2012$

48. Kiesslich T, Berr F, Alinger B, Kemmerling R, Pichler M, Ocker $\mathrm{M}$ and Neureiter D: Current status of therapeutic Targeting of developmental signalling pathways in oncology. Curr Pharm Biotechnol 13: 2184-2220, 2012.

49. Finn RS, Dering J, Ginther C, Wilson CA, Glaspy P, Tchekmedyian N and Slamon DJ: Dasatinib, an orally active small molecule inhibitor of both the src and abl kinases, selectively inhibits growth of basal-type/'triple-negative' breast cancer cell lines growing in vitro. Breast Cancer Res Treat 105: 319-326, 2007. 